\title{
Editorial
}

\section{Fractional Calculus and Its Applications in Applied Mathematics and Other Sciences}

\author{
Santanu Saha Ray, ${ }^{1}$ Abdon Atangana, ${ }^{2}$ S. C. Oukouomi Noutchie, ${ }^{3}$ \\ Muhammet Kurulay, ${ }^{4}$ Necdet Bildik, ${ }^{5}$ and Adem Kilicman ${ }^{6}$ \\ ${ }^{1}$ Department of Mathematics, National Institute of Technology, Rourkela 769008, India \\ ${ }^{2}$ Department of Applied Mathematics, Institute for Groundwater Studies, University of the Free State, Bloemfontein, South Africa \\ ${ }^{3}$ Department of Mathematical Sciences, North-West University, Mafikeng Campus, Mmabatho 2735, South Africa \\ ${ }^{4}$ Department of Mathematics, Yildiz Technical University, 34220 Istanbul, Turkey \\ ${ }^{5}$ Department of Mathematics, Faculty of Art \& Sciences, Celal Bayar University, Muradiye Campus, 45047 Manisa, Turkey \\ ${ }^{6}$ Department of Mathematics and Institute for Mathematical Research, Universiti Putra Malaysia, 43400 Serdang, Selangor, Malaysia
}

Correspondence should be addressed to Santanu Saha Ray; santanusaharay@yahoo.com

Received 9 September 2014; Accepted 9 September 2014; Published 21 December 2014

Copyright (C) 2014 Santanu Saha Ray et al. This is an open access article distributed under the Creative Commons Attribution License, which permits unrestricted use, distribution, and reproduction in any medium, provided the original work is properly cited.

The subject of fractional calculus has applications in diverse and widespread fields of engineering and science such as electromagnetics, viscoelasticity, fluid mechanics, electrochemistry, biological population models, optics, and signals processing. It has been used to model physical and engineering processes that are found to be best described by fractional differential equations. The fractional derivative models are used for accurate modelling of those systems that require accurate modelling of damping. In these fields, various analytical and numerical methods including their applications to new problems have been proposed in recent years. This special issue on "Fractional Calculus and its Applications in Applied Mathematics and Other Sciences" is devoted to study the recent works in the above fields of fractional calculus done by the leading researchers. The papers for this special issue were selected after a careful and studious peer-review process.

Mathematical modelling of real-life problems usually results in fractional differential equations and various other problems involving special functions of mathematical physics as well as their extensions and generalizations in one or more variables. In addition, most physical phenomena of fluid dynamics, quantum mechanics, electricity, ecological systems, and many other models are controlled within their domain of validity by fractional order PDEs. Therefore, it becomes increasingly important to be familiar with all traditional and recently developed methods for solving fractional order PDEs and the implementations of these methods.

The aim of this special issue is to bring together the leading researchers of diverse fields of engineering including applied mathematicians and allow them to share their innovative research work. Analytical and numerical methods with advanced mathematical modelling and recent developments of differential and integral equations of arbitrary order arising in physical systems are included in the main focus of the issue.

Accordingly, various papers on fractional differential equations have been included in this special issue after completing a heedful, rigorous, and peer-review process. The issue contains eight research papers. The issue of robust stability for fractional order Hopfield neural networks with parameter uncertainties is rigorously investigated. Based on the fractional order Lyapunov direct method, the sufficient condition of the existence, uniqueness, and globally robust stability of the equilibrium point is presented. Moreover, the sufficient condition of the robust synchronization between such neural systems with the same parameter uncertainties is proposed owing to the robust stability analysis of its synchronization error system. In addition, for different 
parameter uncertainties, the quasi-synchronization between the classes of neural networks is investigated with linear control. And the quasi-synchronization error bound can be controlled by choosing the suitable control parameters. Moreover, robust synchronization and quasi-synchronization between the classes of neural networks are discussed. The authors have discussed the robust stability and synchronization for the fractional order delayed neural networks (FDNN) with parameter uncertainties.

Some selected wavelet methods have been investigated in order to analyze their accuracy and efficiency with regard to solving linear and nonlinear fractional differential equations. The authors have discussed the challenges faced by researchers in this field and emphasize the importance of interdisciplinary effort for advancing the study on various wavelets in order to solve differential equations of arbitrary order. Several wavelet methods such as Haar wavelet method, cubic B-spline wavelet method, Legendre wavelet method, Legendre multiwavelet method, and Chebyshev wavelet method have been examined for solving fractional differential equations. The Legendre multiwavelet method along with Galerkin method can be applied for providing approximate solutions for initial value problems of fractional nonlinear partial differential equations. Using these wavelet methods the fractional differential equations have been reduced to a system of algebraic equations and this system can be easily solved by any usual methods.

The distributed coordination of fractional multiagent systems with external disturbances is also discussed. The state observer of fractional dynamical system is presented, and an adaptive pinning controller is designed for a little part of agents in multiagent systems without disturbances. Based on disturbance observers, the controllers are composited with the pinning controller and the state observer. By applying the stability theory of fractional order dynamical systems, the distributed coordination of fractional multiagent systems with external disturbances can be reached asymptotically.

Two integral operators involving Appell's functions or Horn's function in the kernel are considered. Composition of such functions with generalized Bessel functions of the first kind is expressed in terms of generalized Wright function and generalized hypergeometric series. Many special cases, including cosine and sine function, are also discussed by the authors and some profound consequences have been drawn.

The existence of solutions for a nonlinear boundary value problem of impulsive fractional differential equations is studied with $p$-Laplacian operator. The research of boundary value problems for $p$-Laplacian equations of fractional order has just begun in recent years.

There are very few results about chaos synchronization of the fractional order time-delay chaotic systems available in the literature. Here chaos synchronization of different fractional order time-delay chaotic systems is also considered. Based on the Laplace transform theory, the conditions for achieving synchronization of different fractional order timedelay chaotic systems are analyzed by use of active control technique. To verify the effectiveness and feasibility of the developed method, numerical simulations are provided by the authors. The authors imposed an improved version of Adams-Bashforth-Moulton method.

Another paper contains the analytical solutions of the fractional order partial differential equations with time and space fractional derivatives which have been solved by means of the homotopy decomposition method (HDM). To show the accuracy and efficiency of this method, three test examples have been considered. The evaluations show that the homotopy decomposition method is extremely successful and suitable. The authors claimed that HDM is an influential and professional tool for FPDEs. He also proclaimed that HDM is superior to the ADM, VIM, HAM, and HPM.

The hydrodynamic dispersion equation was generalized using the concept of variational order derivative. The development of partial differential equations such as the advection-dispersion equation (ADE) begins with assumptions about the random behavior of a single particle. When assumptions underlying the ADE are relaxed, a fractional $\mathrm{ADE}$ (FADE) can arise, with a noninteger-order derivative on time or space terms. Fractional ADEs are nonlocal; they describe transport affected by hydraulic conditions at a distance. Space fractional ADEs arise when velocity variations are heavy tailed and describe particle motion that accounts for variation in the flow field over the entire system. The modified equation was numerically solved via the Crank-Nicholson scheme. The stability and convergence of the scheme in this case were presented. The numerical simulations show that the modified equation is more reliable in predicting the movement of pollution in the deformable aquifers than the constant fractional and integer derivatives.

At present, the use of fractional order partial differential equation in real-physical systems is commonly encountered in the fields of science and engineering. The efficient computational tools are required for analytical and numerical approximations of such physical models. The present issue has addressed recent trends and developments regarding the analytical and numerical methods that may be used in the fractional order dynamical systems. Eventually, it may be expected that the present special issue would certainly helpful to explore the researchers with their new arising fractional order problems and elevate the efficiency and accuracy of the solution methods for those problems in use nowadays.

\author{
Santanu Saha Ray \\ Abdon Atangana \\ S. C. Oukouomi Noutchie \\ Muhammet Kurulay \\ Necdet Bildik \\ Adem Kilicman
}




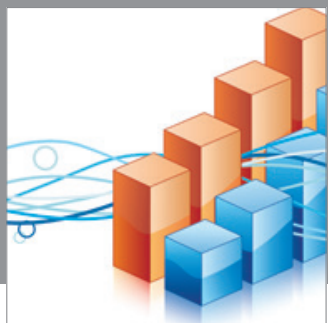

Advances in

Operations Research

mansans

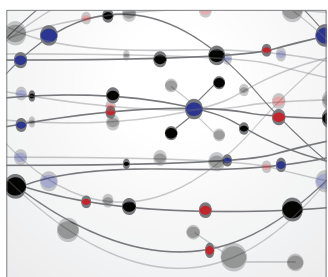

The Scientific World Journal
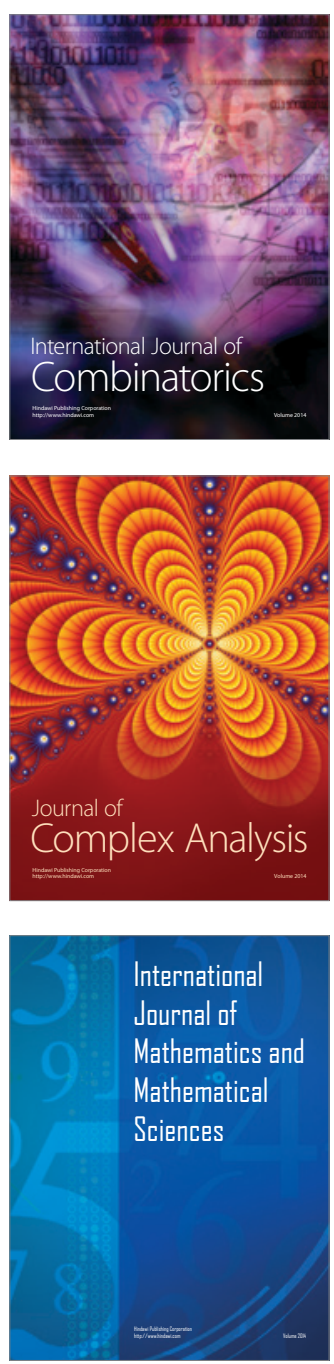
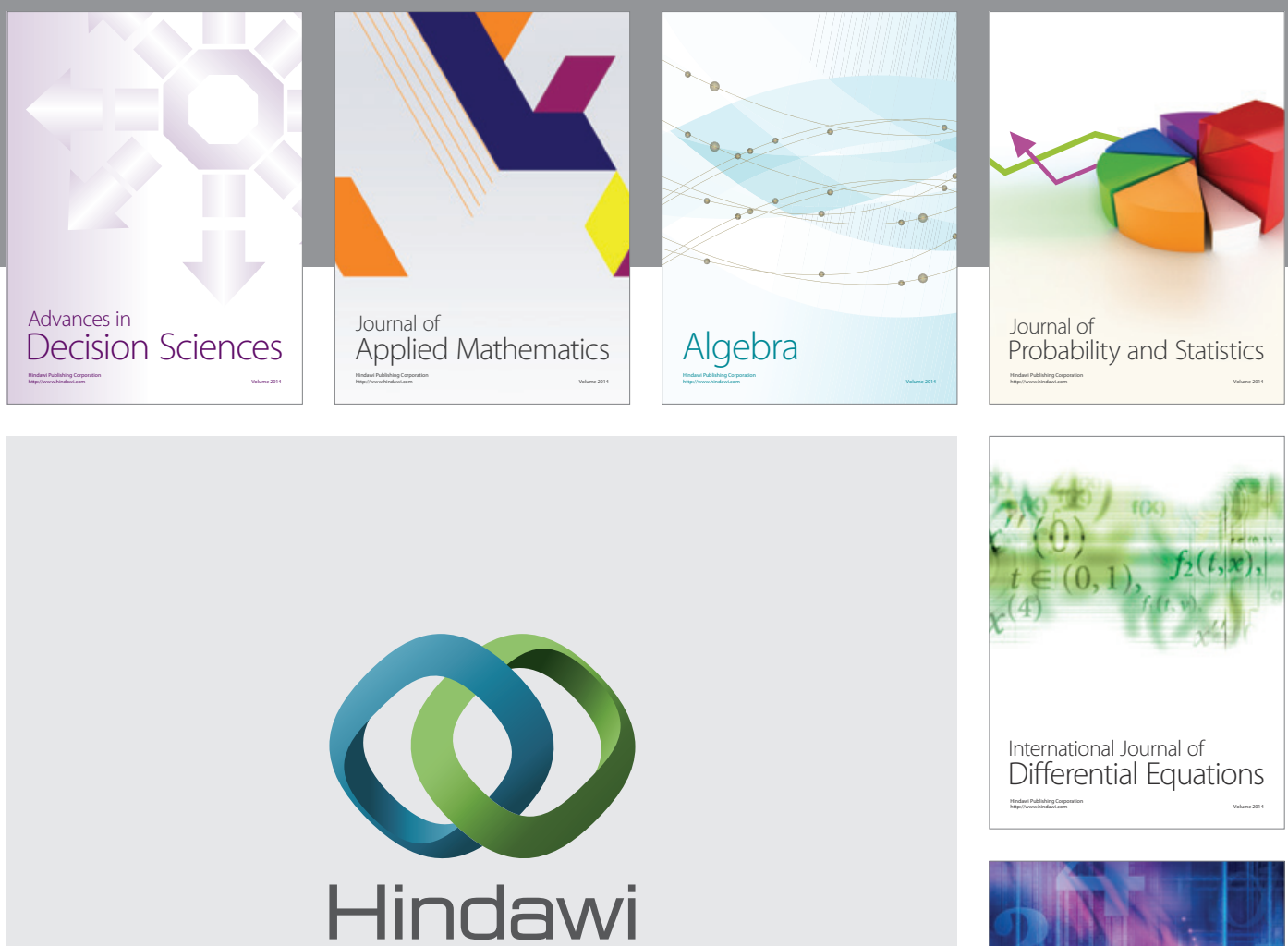

Submit your manuscripts at http://www.hindawi.com
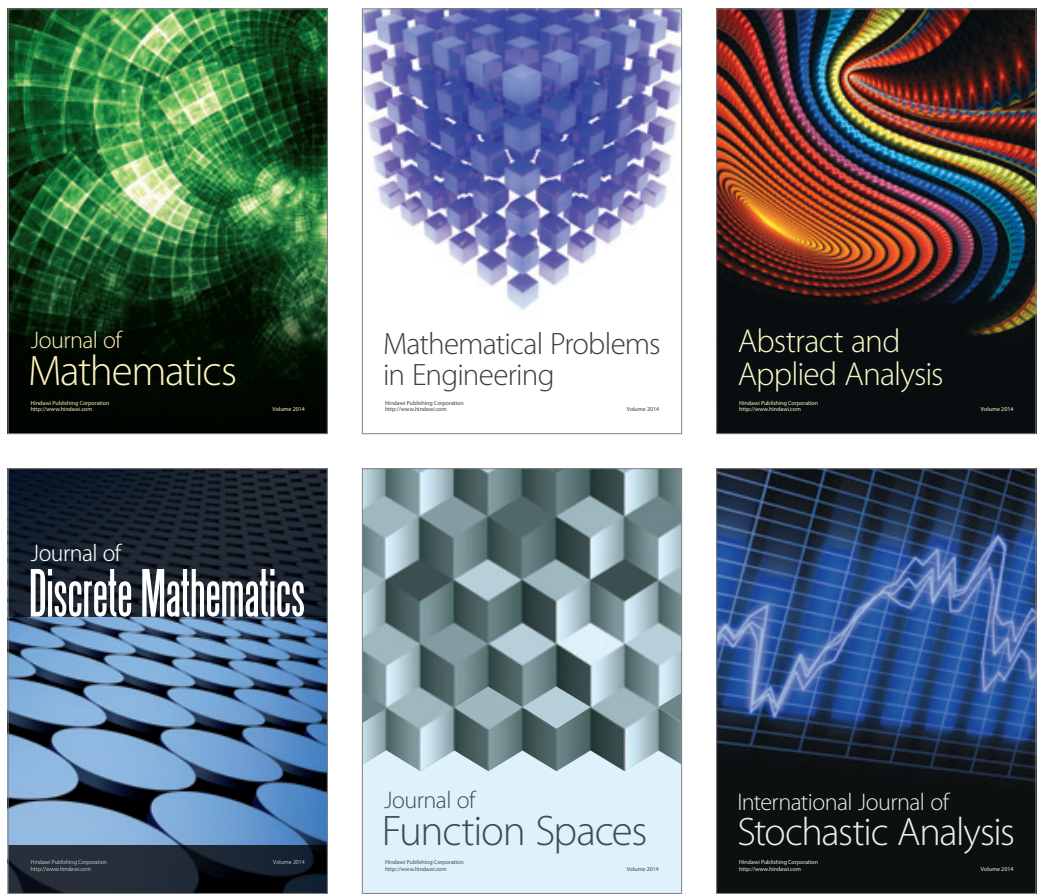

Journal of

Function Spaces

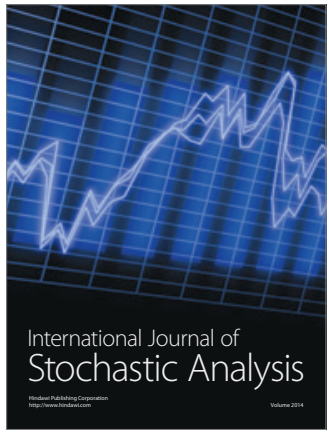

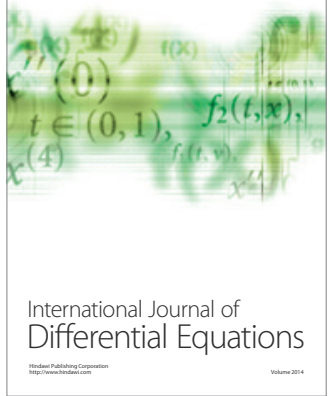
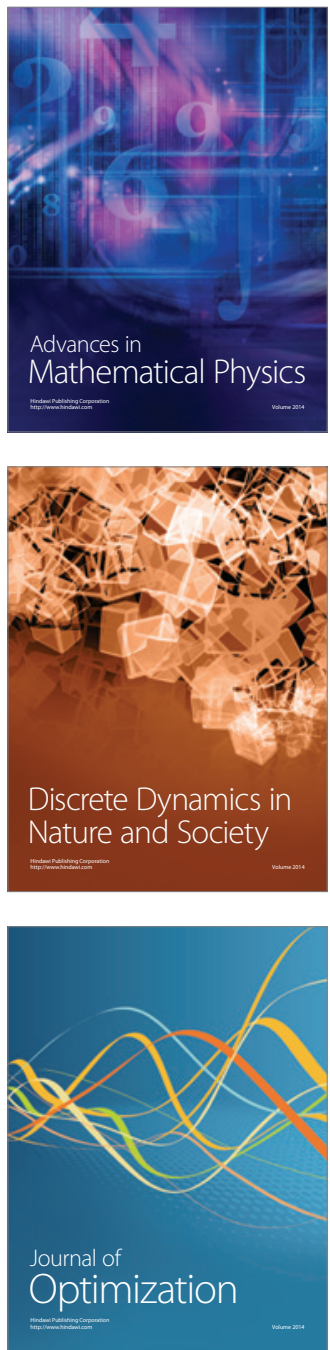